measured 2 hours after the initiation of intravenous PGE1 were slightly increased compared to levels before initiation of intravenous PGE1 ( $\mathrm{p}<0.05)$.

Discussion Although intravenous PGE1 is more effective than oral PGE1 in short term usage, oral PGE1 is also sufficiently effective in keeping the dustus open. For this reason until the intravenous PGE1 is supplied oral PGE1 may be used as an alternative treatment choice. We think that in long term use oral PGE1, which is cheaper and easy to use, could be used instead of intravenous PGE1 without need of admission to hospital and opening intravenous line.

\section{SURGICAL TREATMENT OF PATENT DUCTUS ARTERIOSUS IN PRETERM INFANTS. TEN-YEAR EXPERIENCE WITH LIGATION IN THE NEWBORN INTENSIVE CARE UNIT}

doi:10.1136/archdischild-2012-302724.1129

S Galletti, A Cosentino, A Malavolti, E Macthey, G Faldella. Neonatology and Intensive Care Unit - Sant'Orsola-Malpighi Hospital - University of Bologna, Bologna, Italy

Background and Aim of study Frequently in low-birth-weight infants, ductus arteriosus fails to close spontaneously. This study evaluates the results of surgical ligation of symptomatic PDA in low birth weights preterms.

Methods We reviewed the medical records of all infants undergoing surgical closure of PDA from 2000 to 2010. Demographic data, weight at operation, respiratory assistance pre-operatory, surgical technique to close PDA and outcome were analyzed.

Results Thirty infants underwent surgical closure of PDA in which either indomethacin or ibuprofen treatment had failed or was contraindicated. The mean GA was 27 and the mean birth weight was $752 \mathrm{~g}$. The average weight at operation was $790.5 \mathrm{~g}$. PDA was surgically closed by left thoracotomy using hemoclips.

Postoperative complication occurred in 4 patients, which included intraoperative bleeding (1), pneumothorax (1), lymphatic leak (2). No vocal cord paralysis nor diaphragmatic paralysis were observed. We also registered outcomes related to PDA: broncodisplasia (22), IVH(8), NEC(8), ROP(13). There was no mortality related to surgery.

Conclusion We conclude that surgical closure of hemodynamically significant PDA is safe and effective in preterm low birth weights infants when pharmacological treatment is ineffective or contraindicated. The associated morbidity is minimal and no surgery-related mortality was observed.

\section{EVALUATION OF THE NEONATES WITH CYANOTIC CONGENITAL HEART DEFECTS HOSPITALIZED IN A NICU AT SIX YEARS PERIOD}

doi:10.1136/archdischild-2012-302724.1130

'F Özlü, 'M Satar, ${ }^{2 N}$ Özbarlas, 'H Yapıcıoğlu, 'N Narlı, 'E Kale Çekinmez, 'K Mert. ${ }^{1}$ Neonatology; ${ }^{2}$ Pediatric Cardiology, Çukurova University, Adana, Turkey

Severe congenital heart defects might be symptom free in first days of life. Therefore only half of the congenital heart defects were diagnosed in neonatal period.

In six years period, 86 neonates with the diagnosis of cyanotic congenital heart defects out of 3672 neonates hospitalized in our unit were evaluated. Neonates with the diagnosis of Down syndrome, trisomies and major congenital defects other than heart were excluded from the evaluation. Mean gestational weeks and birth weights of the neonates were 39, 3 (35-40) week and 3128 (1770-4470) gr respectively. The most common pathology of the heart defects was transposition of great arteries (TGA) in 31 neonates $(\% 36)$ and the second common pathology was pulmonary atresia in 17 neonates $(\% 19,8)$. Four of the 31 neonates with TGA had arteriel switch operation and four of them had septostomy procedure, while 23 did not need any invasive procedure. Fourteen of 17 neonates with pulmonary atresia had central or peripheral shunt operations. $51,2 \%$ of the neonates were discharged while $12.8 \%$ of them were discharged due to request of the family. Three of the neonates who had arteriel switch operation and 11 neonates who had shunt operation were discharged.

Early recognition of infants with congenital heart disease that has high mortality and morbidity in neonatal period and implementation of early intervention in patients with ductus-dependent heart defects was considered to have a positive effect on prognosis.

\section{CARDIOVASCULAR AND HORMONAL RESPONSES TO HYPOXIC STRESS IN THE PRETERM PIGLET}

doi:10.1136/archdischild-2012-302724.1131

'YA Eiby, 'MP Staunton, 'LL Wright, 1'2ER Lumbers, 'PB Colditz, 'BE Lingwood. 'UO Centre for Clinical Research, The University of Queensland, Brisbane, OLD; ${ }^{2}$ Mothers and Babies Research Centre, Hunter Medical Research Institute, Newcastle, NSW, Australia

Background and Aims Immature control of the cardiovascular system may be a contributing factor to poor cardiovascular function and associated increases in mortality and morbidity in preterm infants. This study aimed to assess cardiovascular and hormonal responses to a mild hypoxic stress in newborn piglets.

Methods Piglets were delivered by C-section at 97 and 113 days of gestation (term $\approx 115 \mathrm{~d}$ ). An additional preterm group was exposed to maternal glucocorticoid treatment. Changes in mean arterial blood pressure (MAP), skin blood flow and plasma concentrations of adrenalin, noradrenalin, angiotensin II and cortisol were measured in response to acute hypoxia ( $4 \% \mathrm{O}_{2}$ for $20 \mathrm{~min}$ ).

Results Preterm piglets were less likely to exhibit a mature cardiovascular compensatory response to hypoxia (increased MAP and reduced skin blood flow) than term piglets. Plasma adrenalin and noradrenalin concentrations at the end of hypoxia were increased in all groups and were $2-3$ fold higher in preterm pigs than in term pigs $(\mathrm{P}<0.05)$. Plasma cortisol levels were increased at the end of hypoxia in term piglets $(\mathrm{P}<0.05)$ but not in preterm or glucocorticoid exposed preterm piglets. Angiotensin II levels were reduced in glucocorticoid exposed preterm piglets compared to untreated preterm piglets $(\mathrm{P}<0.05)$.

Conclusions The reduced cardiovascular compensatory response to hypoxia in preterm pigs is not the result of reduced plasma levels of catecholamines during hypoxia. Low levels of angiotensin II in glucocorticoid exposed preterm piglets may contribute to poor control of skin blood flow during hypoxia.

\section{THE EFFECT OF PLATELET COUNT ON SUCCESSFUL MEDICAL CLOSURE OF PATENT DUCTUS ARTERIOSUS}

doi:10.1136/archdischild-2012-302724.1132

P Nath, M Avondo, R Roy. Neonatal Unit, Norfolk and Norwich University Hospital NHS Trust, Norwich, UK

Background and Aim Patent Ductus Arteriosus (PDA) is a shunt vessel connecting the pulmonary artery and the aorta. It usually closes after birth, but persistence of PDA in preterm neonates is a major cause of morbidity and mortality. A recent study suggested that platelet counts are likely to contribute to PDA closure in humans.

We aim to investigate relationship between platelet counts and closure of PDA after medical treatment with Indomethacin or I buprofen.

Methods All preterm infants born $\leq 32$ weeks and birth weight $\leq 1500$ grams born between 01 January 2008 and 31 December 2010 were included. Retrospective data was collected from the case records, SEND neonatal database and laboratory result systems. 
Results A total of 58 babies were identified with 64 episodes of medical treatment for PDA with Indomethacin or Ibuprofen. Mean gestational age was 25.5 weeks and birth weight of 737.1 grams. Mean age at treatment was 26.7 weeks with pre-treatment PDA size of $1.5-6 \mathrm{~mm}$ and platelet count of $34-602 \times 10^{9} / \mathrm{L}$.

Overall 73\% (47/64) were treated with high dose and 11\% (7/64) with low dose Indomethacin. 30\% (19/64) PDA closed post treatment, $62 \%$ (40/64) remained open.

Out of 19 successful closures 7 had initial platelet counts of $>150$ (Odds Ratio 0.31, 95\% Confidence Interval) and out of 40 unsuccessful closures 26 had initial platelet counts of $>150$ (Odds Ratio $3.18,95 \%$ Confidence Interval).

Conclusions Success of PDA closure after medical treatment was not related to the platelet counts in our study group.

\section{ANTENATAL VS POSTNATAL DETECTION OF MAJOR CONGENITAL HEART DISEASE IN A LARGE DISTRICT- GENERAL HOSPITAL IN UK; A SIX-YEAR REVIEW}

doi:10.1136/archdischild-2012-302724.1133

'S Rajendran, 'A Shastri, ${ }^{2} \mathrm{~K}$ Annam. ' Colchester General Hospital, Colchester Hospital University; ${ }^{2}$ Colchester General Hospital, Colchester Hospital University Foundation Trust, Colchester, UK

Background and Aims Major congenital heart disease (CHD) is defined as CHD that needs operative or catheter based intervention in the first year of life. National institute of clinical excellence (NICE) in March 2008 recommended screening of outflow-tracts in addition to four-chamber view as part of the anomaly scan to improve CHD detection rates. We aimed to examine the clinical spectrum of antenatally and postnatally diagnosed major CHD in our institute pre- and post-introduction of NICE guideline.

Methods This is a retrospective review over six years from Jan 2006 to Dec 2011. Data was obtained from antenatal records, patient's clinical and electronic records.

Results A total of 74 babies had major CHD diagnosed out of which 37 (50\%) were diagnosed antenatally. Antenatal diagnosis pre- and post- NICE guidelines were 12/29 (41\%)and 25/45 (55\%) respectively as also termination of pregnancies with critical CHD doubled. Common postnatal presentations included cardiovascular collapse $4(11 \%)$, cyanosis $8(22 \%)$, murmurs $12(32 \%)$, heart failure $5(13.5 \%)$, faltering growth $5(13.5 \%) .4$ babies were critically ill with severe acidosis and 3 needed intubation and ventilation prior to transfer to a tertiary unit. Median age at intervention for duct dependent lesions was 10 days and other major CHD was 5 months. Conclusions Although antenatal screening methods have improved, a large proportion of babies with CHD are still undetected. Routine Pulse-oximetry screening has proved to be an effective screening adjunct in four large studies and hence should be considered as part of early neonatal examination.

\section{PROTEASE-ACTIVATED RECEPTOR (PAR)-MEDIATED CONTRACTION OF THE CHICKEN DUCTUS ARTERIOSUS}

doi:10.1136/archdischild-2012-302724.1134

K Kartal, J Meekels, R Mohammed, S van der Sterren, E Villamor. Pediatrics, Maastricht University Medical Center, Maastricht, The Netherlands

Background and Aims PARs belong to a family of $G$ protein- coupled receptors, thus mediating the cellular effects of proteinases. PAR1 and PAR2 have been shown to be involved in regulating vascular tone. Thrombin activates PAR1, whereas trypsin activates PAR1 and PAR2. Our aim was to evaluate the functional presence of PAR1 and PAR2 in the ductus arteriosus (DA).

Methods We investigated, using wire myography, the mechanical responses induced by thrombin $(0.1$ to $3 \mathrm{U} / \mathrm{mL})$, trypsin $(0.1$ to 30 $\mathrm{U} / \mathrm{mL}$ ), the PAR1-activating peptide TFLLR- $\mathrm{NH}_{2}(1$ to $100 \mu \mathrm{mol} / \mathrm{L})$ and the PAR2-activating peptide SLIGRL- $\mathrm{NH}_{2}(0.1$ to $10 \mu \mathrm{mol} / \mathrm{L})$ in DA rings from 15-, 19-, and 21-d chicken embryos.

Results Thrombin, trypsin, and TFLLR- $\mathrm{NH}_{2}$, all caused concentration-dependent contraction of the pulmonary side of chicken DA. These contractions were not observed in the aortic side of the DA, in the femoral artery or in the pulmonary artery. Thrombin-, trypsin- and TFLLR- $\mathrm{NH}_{2}$-induced contractions were endotheliumindependent but markedly impaired by the elimination of calcium from the external medium. The contraction evoked by thrombin and trypsin increased between day 15 and 19 of incubation and was not affected by oxygen tension. SLIGRL- $\mathrm{NH}_{2}(\geq 10 \mu \mathrm{mol} / \mathrm{L})$, evoked endothelium-dependent relaxation of the DA.

Conclusions PARs are functionally present in the chicken DA but not in other vascular tissues. Recent studies demonstrate that loss of platelet number or function leads to defective DA closure. We speculate that the role of platelets in DA closure might be partially mediated through the PAR-mediated vasoactive effects of thrombin.

\section{CORONARY ARTERY ANATOMY PRIOR TETRALOGY OF FALLOT SURGERY}

doi:10.1136/archdischild-2012-302724.1135

${ }^{1} \mathrm{~S}$ Mesihovic-Dinarevic, 'Z Begic, ${ }^{1} \mathrm{~A}$ Kadic, ${ }^{1} \mathrm{M}$ Halimic, ${ }^{2} \mathrm{~S}$ Pandur, ${ }^{2} \mathrm{H}$ Vila, ${ }^{2} \mathrm{E}$ Omerbasic, II Poplata. 'Peadiatric Clinic, Clinical Centre University of Sarajevo; ${ }^{2}$ Heart Centre, Clinical Centre University of Sarajevo, Sarajevo, Bosnia-Herzegovina

Intoduction Anomalous coronary artery disease is common among patients with Tetralogy of Fallot (ToF). The need to define the morfohaemodynamics of TOF is the priority in echo laboratory prior TOF surgery.

Aim To evaluated coronary artery anatomy pre and intraoperatively by imaging technique-echocardiograhy and to establish frequency of congenital coronary arteries anomalies in patients with tetralogy of Fallot, as a plan of surgical approach in these patients.

Methods Consecutive retrospective analysis of echocardiography data patients with tetralogy of Fallot and surgical protocols of total corrections of Tetralogy of Fallot was performed.

Results During period from 01.01.2010 till 01.01.2011. at Paediatric clinic CCU Sarajevo Out pts Cardiology department 3577 pts were evaluated, $70 / 1.9 \%$ /pts with CHD and $13 / 70$ with ToF (18\%), age 25 monhts (9-60), 7 girls (53.8\%). First Group had simple ToF/n-10/and Second Group had Pentalogia Fallot (n-3 pts). Anatomical correction was performed in all pts in Sarajevo by foregin an local team. Preoperativey $2 / 15.4 \%$ /pts with ToF had anmalous origin of anterior interventricular branch of RCA crossing RVOT which was as well confirmed intraoperatively. The surgical approcah was based upon the preoperativelly echo findings, so these 2 pts had RVOT-PA graft construction. All pts from the Second Group had normal anatomy of coronary arteries pre- and intraoperatively. Conclusion Non invasive transtoracic echocardiography (TTE) is very usefull tool in deliniation of coronary artery anatomy as a part of surgical approach plan in patients with TOF which defenitly could decrease the risk of surgery.

\section{MORPHOHEMODYNAMIC MONITORING OF PULMONARY ARTERY IN PREMATURE INFANTS}

doi:10.1136/archdischild-2012-302724.1136

E Hadimujic-Konjalic, S Mesihovic-Dinarevic, S Terzic. Pediatric, Clinic Center Sarajevo, Sarajevo, Bosnia-Herzegovina

Introduction Two-dimensional echocardiography is increasingly used in Neonatal intensive care departments in order to test structure and function of the hearth because a definite diagnosis in this period is very difficult if solely based on physical examination. 